\title{
A two-microRNA-based signature predicts first-line chemotherapy outcomes in advanced colorectal cancer patients
}

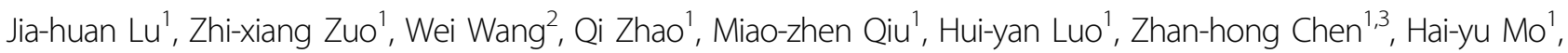 \\ Feng Wang ${ }^{1}$, Dong-dong Yang ${ }^{1}$, Yun Wang ${ }^{1}$, Xiao-li Wei ${ }^{1}$, Qi-nian Wu' ${ }^{1}$, Huai-qiang Ju', Rui-hua Xu ${ }^{1}$ and \\ Zhao-lei Zeng (10 ${ }^{1}$
}

\begin{abstract}
Prognostic and predictive markers are needed to predict the clinical outcomes of patients with advanced colorectal cancer (CRC) who receive standard first-line treatments. We performed a prospective cohort study in advanced CRC patients to identify a miRNA signature that could predict the benefit of receiving first-line chemotherapy for these patients. Twenty-one paired tumours and adjacent normal tissues were collected from advanced CRC patients and analysed by miRNA microarrays. Between tumour and normal tissues, 33 miRNAs were differentially expressed and was confirmed by qRT-PCR from another group of 67 patients from a prospective cohort study. A two-miRNA-based signature was obtained using the LASSO Cox regression model based on the association between the expression of each miRNA and the PFS of individual patients. Internal and external validation cohorts, including 40 and 44 patients with advanced CRC, respectively, were performed to prove the prognostic and predictive value of this signature. A signature was built based on two miRNAs, miR-125b-2-3p and miR-933. CRC patients were classified into low- and high-risk groups for disease progression based on this tool. The patients with low risk scores generally had better PFS than those with high risk scores. In the training set, the median PFS in the low- and high-risk groups were 12.00 and 7.40 months, respectively. In the internal validation set, the median PFS in the low- and high-risk groups were 9.90 and 5.10 months, respectively. In the external validation set, the median PFS in the low- and high-risk groups were 9.90 and 6.40 months, respectively. Furthermore, we detected miR-125b-2-3p associated with CRC cell sensitivity to first-line chemotherapy. Our two-miRNA-based signature was a reliable prognostic and predictive tool for tumour progression in patients with advanced CRC, and might be able to predict the benefit of receiving standard first-line chemotherapy in CRC.
\end{abstract}

\section{Introduction}

Colorectal cancer (CRC) is the third most common cancer diagnosed in both men and women and is the fourth leading cause of cancer-related death worldwide ${ }^{1,2}$. An

\footnotetext{
Correspondence: Z.-I. Zeng (zengzhl@sysucc.org.cn)

${ }^{1}$ State Key Laboratory of Oncology in South China, Collaborative Innovation Center for Cancer Medicine, Sun Yat-sen University Cancer Center, 510060

Guangzhou, China

${ }^{2}$ Foshan First People's Hospital, 528000 Foshan, China

Full list of author information is available at the end of the article.

These authors contributed equally: Jia-huan Lu, Zhi-xiang Zuo, Wei Wang

Edited by E. Sayan
}

estimated 1.4 million new cases of CRC are clinically diagnosed globally, resulting in 693,900 patient deaths from CRC in $2012^{3}$. Over $20 \%$ of CRC cases are diagnosed at stage IV, an advanced stage, and higher percentages have been reported in developing countries ${ }^{4,5}$. Chemotherapy is one of the most common treatments for advanced CRC patients. FOLFOX (5-fluorouracil (5-FU), leucovorin and oxaliplatin)/XELOX (capecitabine and oxaliplatin) and FOLFIRI (5-FU, leucovorin and irinotecan) are the first-line chemotherapeutic strategies for advanced $\mathrm{CRC}^{6}$. However, these similar chemotherapeutic strategies result in varying

\section{(c) 2018 The Author(s)}

\footnotetext{
(c) (i) Open Access This article is licensed under a Creative Commons Attribution 4.0 International License, which permits use, sharing, adaptation, distribution and reproduction cc) in any medium or format, as long as you give appropriate credit to the original author(s) and the source, provide a link to the Creative Commons license, and indicate if changes were made. The images or other third party material in this article are included in the article's Creative Commons license, unless indicated otherwise in a credit line to the material. If material is not included in the article's Creative Commons license and your intended use is not permitted by statutory regulation or exceeds the permitted use, you will need to obtain permission directly from the copyright holder. To view a copy of this license, visit http://creativecommons.org/licenses/by/4.0/.
} 
responses and different clinical outcomes in advanced $\mathrm{CRC}^{7-9}$. Moreover, primary and acquired drug resistance remain significant challenges to achieving satisfactory treatment effects. Thus, identifying effective biomarkers is essential for improving the prediction of treatment responses and guiding treatment decisions in advanced CRC patients.

MicroRNAs (miRNAs) are an abundant class of small non-coding RNAs that typically induce gene silencing or mRNA degradation by binding to the target sites in the $3^{\prime}$ untranslated regions (UTRs) of their targeted mRNAs ${ }^{10,11}$. MiRNAs are involved in varied biological and pathophysiological processes, such as the cell cycle, cell differentiation, proliferation, apoptosis, etc. ${ }^{12-14}$. Apart from their important roles in biological processes, miRNAs also participate in a wide range of diseases, including many types of cancers ${ }^{15,16}$. Accordingly, miRNAs are studied as candidates for diagnostic and prognostic biomarkers and predictors of drug responses ${ }^{17,18}$. Several studies have demonstrated the roles of miRNAs as chemotherapeutic predictors in $\mathrm{CRC}^{19-21}$. Compared to a single biomarker, integrating multiple biomarkers into a single model can significantly improve the predictive value $^{22,23}$.

In the present prospective observational study, we aimed to exploit and validate a multiple-miRNA-based signature using the least absolute shrinkage and selection operator (LASSO) Cox regression model to predict the progression-free survival (PFS) and response to controversial chemotherapeutic strategies in advanced CRC patients. We assessed the prognostic and predictive value of this signature in the training set, and then we validated this signature in two validation cohorts. Thus, our study identifies an effective prognostic and predictive biomarker signature for advanced CRC patients receiving standard first-line treatments.

\section{Results}

\section{Clinicopathological characteristics of patients}

A total of 151 patients with histopathologically and clinically diagnosed advanced CRC were enrolled in the present study according to the selection criteria. The patients from SYSUCC were included and randomly assigned to the training set (67 patients) and internal testing set (40 patients), and another 44 patients from FPHFS were recruited as the external validation set. The clinicopathological characteristics of the patients in each set are shown in Table 1.

Building a two-miRNA-based prognostic classifier with an integrated marker selection approach and Cox regression

Twenty-one pairs of tumour and adjacent normal tissues from advanced CRC patients were used in the microarray analysis. To obtain the most significant
miRNAs for classifying high- and low-risk progressive patients, we applied an integrated marker selection method, consisting of differential expression analysis, univariable Cox regression analysis and LASSO bagging variable selection. The flowchart of microRNA filtration is illustrated in Supplementary Fig. S1. First, using the microarray data matrix, differential expression analysis was performed with the limma package between tumour and normal tissues ${ }^{24}$. A total of 178 upregulated miRNAs and 231 down-regulated miRNAs were identified in the tumour samples. Then, with the same data set, Cox regression analyses with a Wald threshold of $p<0.1$ were run individually to screen the progression-related markers. A total of 33 miRNAs were identified as differentially expressed between the tumour and adjacent normal samples and were correlated with the patients' PFS $(p<$ 0.1, Kaplan-Meier method). Hierarchical clustering showed that the 21 pairs of tumour and adjacent normal tissues were separated into two discrete groups (Fig. 1a). The expression of these 33 miRNAs in tumour and adjacent normal tissues was validated by qRT-PCR (Supplementary Fig. S2), confirming the differential expression of these miRNAs between tumour and adjacent normal tissues.

To further narrow down a miRNA-based signature for patients with advanced CRC, we sequentially applied a LASSO bagging method on the qRT-PCR dataset from the training set. Briefly, the LASSO method is commonly used for regression with high-dimensional predictors, and its extended model has been widely applied to the Cox proportional hazard regression model for the analysis of high-dimensional survival data ${ }^{25}$. For LASSO bagging, bootstrap samples of the data (sampling with replacement with a sample size equal to that of the original data) were used to perform the entire LASSO Cox procedure, including optimal tuning parameter selection (also called $\lambda)$ and variable selection steps. In the present analysis, we obtained 1000 resamples and used different optimal $\lambda$ parameters selected via 1-SE (standard error) criteria for each resample. We then calculated the RMIP for each miRNA. The RMIP is a measure of how likely a given miRNA is selected by the LASSO procedure if the dataset is perturbed. We observed continuing sharp decreases of RMIP until the third miRNA miR-5571-5p as ordered by the RMIP of each miRNA. As a result, two miRNAs, miR-125b-2-3p and miR-933, were selected for building a prognostic classifier (Fig. 1b). Additionally, a two-variable Cox regression analysis was performed on the same data to determine the coefficients of the two miRNAs, which were -0.259 for miR-125b-2-3p and 0.092 for miR-933.

Using a two-variable Cox regression model, we obtained the risk score for each patient (risk score $=$ $0.092 \times \mathrm{miR}-933-0.259 \times \mathrm{miR}-125 \mathrm{~b}-2-3 \mathrm{p}$ ). Based on the signature, the risk score for each patient in the 
Table 1 Clinicopathologic characteristics of two sets of CRC patients according to the two-miRNAs signature

\begin{tabular}{|c|c|c|c|c|c|c|c|c|c|c|c|c|}
\hline \multirow[b]{2}{*}{ Characteristics } & \multirow[b]{2}{*}{ No. } & \multicolumn{3}{|c|}{ Training set $(n=67)$} & \multirow[b]{2}{*}{ No } & \multicolumn{3}{|c|}{ Internal testing set $(n=40)$} & \multirow[b]{2}{*}{ No. } & \multicolumn{3}{|c|}{$\begin{array}{l}\text { Independent validation set } \\
(n=44)\end{array}$} \\
\hline & & $\begin{array}{l}\text { Low risk } \\
\text { (\%) }\end{array}$ & $\begin{array}{l}\text { High risk } \\
(\%)\end{array}$ & $p$ value & & $\begin{array}{l}\text { Low risk } \\
(\%)\end{array}$ & $\begin{array}{l}\text { High risk } \\
\text { (\%) }\end{array}$ & $p$ value & & $\begin{array}{l}\text { Low risk } \\
\text { (\%) }\end{array}$ & $\begin{array}{l}\text { High risk } \\
(\%)\end{array}$ & $p$ value \\
\hline Gender & & & & 0.68 & & & & 0.35 & & & & 0.60 \\
\hline Male & 42 & $18(42.9)$ & $24(57.1)$ & & 25 & $17(68.0)$ & $8(32.0)$ & & 27 & $18(66.7)$ & $9(33.3)$ & \\
\hline Female & 25 & $12(48.0)$ & $13(52.0)$ & & 15 & $8(53.3)$ & $7(46.7)$ & & 17 & $10(58.8)$ & $7(41.2)$ & \\
\hline Age & & & & 0.09 & & & & 0.62 & & & & 0.16 \\
\hline$<60$-year-old & 41 & $15(36.6)$ & $26(63.4)$ & & 22 & $13(68.4)$ & $9(31.6)$ & & 28 & $20(71.4)$ & $8(28.6)$ & \\
\hline$\geq 60$-year-old & 26 & $15(57.7)$ & $11(42.3)$ & & 18 & $12(66.7)$ & $6(33.3)$ & & 16 & $8(50.0)$ & $8(50.0)$ & \\
\hline Tumour location & & & & 0.14 & & & & 0.67 & & & & 0.72 \\
\hline Colon & 50 & $25(50.0)$ & $25(50.0)$ & & 25 & $15(60.0)$ & $10(40.0)$ & & 29 & $19(65.5)$ & $10(34.5)$ & \\
\hline Rectum & 17 & $5(29.4)$ & $12(70.6)$ & & 15 & $10(66.7)$ & $5(33.3)$ & & 15 & $9(66.7)$ & $6(33.3)$ & \\
\hline Tumour grade & & & & 0.35 & & & & 0.55 & & & & 0.70 \\
\hline Low & 6 & $1(16.7)$ & $5(83.3)$ & & 3 & $1(33.3)$ & $2(66.7)$ & & 5 & $4(80.0)$ & $1(20.0)$ & \\
\hline Median & 57 & $27(47.4)$ & $30(52.6)$ & & 34 & $22(64.7)$ & $12(35.3)$ & & 38 & $23(60.5)$ & $15(39.5)$ & \\
\hline High & 4 & $2(50.0)$ & $2(50.0)$ & & 3 & $2(66.7)$ & $1(33.3)$ & & 1 & $1(100.0)$ & $0(0.0)$ & \\
\hline Metastatic location & & & & 0.68 & & & & 0.57 & & & & 0.63 \\
\hline Liver & 42 & $18(42.9)$ & $24(57.1)$ & & 30 & $18(60.0)$ & $12(40.0)$ & & 34 & $21(61.8)$ & $13(38.2)$ & \\
\hline Others & 25 & $12(48.0)$ & $13(52.0)$ & & 10 & $7(70.0)$ & $3(30.0)$ & & 10 & $7(70.0)$ & $3(30.0)$ & \\
\hline Metastatic type & & & & 0.82 & & & & 0.43 & & & & 0.91 \\
\hline Synchronous & 50 & $22(44.0)$ & $28(56.0)$ & & 27 & $18(66.7)$ & $9(33.3)$ & & 27 & $17(63.0)$ & $10(27.0)$ & \\
\hline Metachronous & 17 & $8(47.1)$ & $9(52.9)$ & & 13 & $7(53.8)$ & $6(46.2)$ & & 17 & $11(64.7)$ & $6(35.3)$ & \\
\hline Chemotherapy strategy & & & & 0.69 & & & & 0.28 & & & & 0.91 \\
\hline FOLFOX & 55 & $24(43.6)$ & $31(56.4)$ & & 31 & $18(58.0)$ & $13(42.0)$ & & 27 & $17(63.0)$ & $10(27.0)$ & \\
\hline FOLFIRI & 12 & $6(50.0)$ & $6(50.0)$ & & 9 & $7(77.8)$ & $2(22.2)$ & & 17 & $11(64.7)$ & $6(35.3)$ & \\
\hline $\begin{array}{l}\text { Response for } \\
\text { chemotherapy }\end{array}$ & & & & $<0.001$ & & & & $<0.001$ & & & & $<0.001$ \\
\hline$C R+P R$ & 23 & $15(65.2)$ & $8(34.8)$ & & 10 & $9(90.0)$ & $1(10.0)$ & & 11 & $6(54.5)$ & $5(45.5)$ & \\
\hline SD & 31 & $11(35.5)$ & $20(64.5)$ & & 21 & $13(61.9)$ & $8(38.1)$ & & 21 & $17(81.0)$ & $4(19.0)$ & \\
\hline PD & 13 & $4(30.8)$ & $9(69.2)$ & & 9 & $3(33.3)$ & $6(66.7)$ & & 12 & $5(41.7)$ & $7(58.3)$ & \\
\hline
\end{tabular}

training cohort was determined. The median value of the risk score in the training set was taken as the cut-off value (0.022), and all the patients were then assigned to low- or high-risk groups based on their risk scores.

Post hoc statistical power analysis was used to assess adequacy of research design. The post hoc statistical power analysis showed an adequate power of $88.3 \%$ in training cohort and $98.4 \%$ for all samples.

\section{Prognostic value of the miRNA signature}

To examine whether the signature was associated with PFS, we applied multivariate Cox proportional hazards regression analyses in the training, internal testing and external validation sets (Table 2). The patients with high risk scores generally displayed worse PFS than those with lower risk scores in the training set; the median PFS was 12.00 months (95\% CI 9.89-14.11 m) for the low-risk group and 7.40 months $(95 \%$ CI $6.56-8.24 \mathrm{~m}$ ) for the high-risk group $(p<0.001)$. To confirm the prognostic accuracy of the two-miRNA-based signature, we conducted analyses using the internal testing and external validation sets. Similar results were observed in these two sets. In the internal testing set, the median PFS was 9.90 months (95\% CI 6.89-12.91 m) for the low-risk 


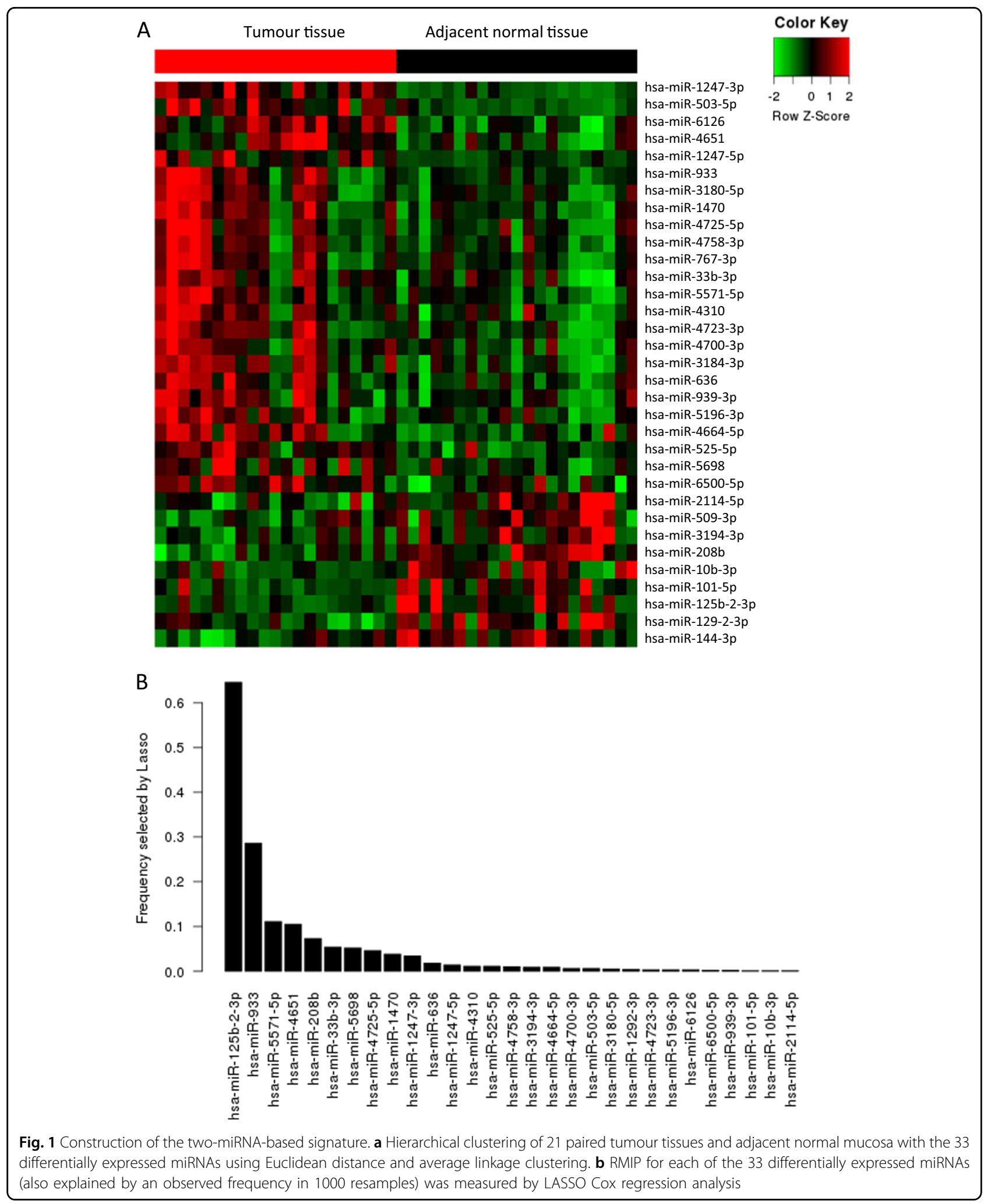

group and 5.10 months (95\% CI 3.57-6.63 m) for the high-risk group $(p<0.001)$. In the external validation set, the median PFS was 9.90 (95\% CI 7.33-12.47 m) for the low-risk group and 6.40 months (95\% CI $4.87-7.93 \mathrm{~m})$ for the high-risk group $(p=0.002)$. For all the patients, the median PFS in the low- and high-risk groups was 11.30 
Table 2 Multivariate Cox proportional hazards regression analysis of the clinicopathologic characteristics and integrated microRNA signature with PFS

\begin{tabular}{|c|c|c|c|c|c|c|}
\hline & \multicolumn{2}{|c|}{ Training set $(n=67)$} & \multicolumn{2}{|c|}{ Internal testing set $(n=40)$} & \multicolumn{2}{|c|}{$\begin{array}{l}\text { Independent validation } \\
\text { set }(n=44)\end{array}$} \\
\hline & $\mathrm{HR}(95 \% \mathrm{Cl})$ & $p$ & $\mathrm{HR}(95 \% \mathrm{Cl})$ & $p$ & $\mathrm{HR}(95 \% \mathrm{Cl})$ & $p$ \\
\hline Gender & $1.034(0.584-1.830)$ & 0.910 & $1.410(0.661-3.007)$ & 0.374 & $0.898(0.456-1.769)$ & 0.757 \\
\hline Age (<60-year-old vs $\geq 60$ year-old) & $0.771(0.441-1.347)$ & 0.361 & $0.667(0.269-1.657)$ & 0.383 & $0.726(0.370-1.426)$ & 0.353 \\
\hline Tumour location (colon vs rectum) & $1.185(0.651-2.160)$ & 0.578 & $1.084(0.505-2.325)$ & 0.836 & $1.388(0.669-2.880)$ & 0.379 \\
\hline Tumour grade (low vs median/high) & $0.531(0.232-1.215)$ & 0.134 & $2.743(1.020-7.383)$ & 0.046 & $1.576(0.475-5.229)$ & 0.457 \\
\hline Metastatic location (without vs with liver metastasis) & $1.004(0.569-1.770)$ & 0.989 & $2.446(0.908-6.587)$ & 0.077 & $2.086(0.842-5.171)$ & 0.112 \\
\hline Metastatic site number (one vs more than one) & $1.601(1.119-2.290)$ & 0.010 & $1.192(0.792-1.792)$ & 0.400 & $1.853(1.070-3.210)$ & 0.028 \\
\hline Metastatic type (synchronous vs metachronous) & $0.617(0.315-1.209)$ & 0.159 & $1.099(0.481-2.512)$ & 0.823 & $1.235(0.624-2.444)$ & 0.544 \\
\hline Chemotherapy strategy (FOLFOX vs FOLFIRI) & $1.151(0.577-2.299)$ & 0.690 & $0.756(0.304-1.877)$ & 0.546 & $0.741(0.372-1.478)$ & 0.395 \\
\hline Response for chemotherapy (CR + PR + SD vs PD) & $4.608(2.337-9.083)$ & $<0.001$ & $12.033(4.008-36.130)$ & $<0.001$ & $2.802(1.276-6.152)$ & 0.010 \\
\hline miR-125b-2-3p (low vs high expression) & $0.526(0.29-0.952)$ & 0.034 & $0.443(0.203-0.969)$ & 0.041 & $0.487(0.244-0.971)$ & 0.041 \\
\hline miR-933 (low vs high expression) & $0.875(0.490-1.565)$ & 0.653 & $0.834(0.434-1.941)$ & 0.340 & $0.616(0.309-1.229)$ & 0.169 \\
\hline miRNA signature (low vs high risk) & $2.817(1.584-5.009)$ & $<0.001$ & $7.797(2.525-24.089)$ & $<0.001$ & $3.153(1.444-6.887)$ & 0.004 \\
\hline
\end{tabular}

(95\% CI $9.36-13.24 \mathrm{~m})$ and 7.20 months $(95 \% \mathrm{CI}$ $6.69-7.81 \mathrm{~m})$, respectively $(p<0.001)$ (Fig. $2 \mathrm{a})$. The progressive rate was $13.33 \%$ (4/30) for the low-risk group and $24.32 \%(9 / 37)$ for the high-risk one in the training set $(p<$ $0.001) ; 12.00 \%(3 / 25)$ for the low-risk group and $40.00 \%$ $(6 / 15)$ for the high-risk group in the internal testing set $(p$ $<0.001)$; and $17.86 \%(5 / 28)$ vs. $43.75 \%$ (7/16), respectively, in the external validation set $(p<0.001)$. For all patients, the progressive rate was $14.63 \%(12 / 82)$ for the low-risk group and $31.88 \%(22 / 69)$ for the high-risk one, respectively ( $p<0.001$; Fig. $2 \mathrm{~b})$.

To further assess the prognostic value of the twomiRNA signature on risk score prediction, we calculated the time-dependent receiver operating characteristic (ROC) in the three cohorts respectively. The two-miRNAbased signature showed significantly higher prognostic value than any other single clinicopathological risk factor $(p<0.05)$ (Fig. 3).

\section{Predictive value of the signature for chemotherapy sensitivity}

We used multivariate Cox proportional hazards regression analysis to test the predictive value of the signature for first-line chemotherapy sensitivity in advanced CRC cases (Table 3). The interactions between the risk status and patient response to chemotherapy were analysed. The interactions between these were dramatically significant in the training set ( $\mathrm{HR}$ 3.644; 95\% CI $1.528-8.690 ; p=0.004)$, in the internal testing set (HR 8.426; 95\% CI 2.592-27.390; $p<0.0001)$, and in the external validation set (HR 2.653; 95\% CI 1.031-6.829; $p=0.043)$. These data suggested that this two-miRNAbased signature might be valuable for predicting the benefit of standard first-line chemotherapy in advanced CRC.

\section{Drug effects of the two microRNAs}

To explore the effect of miR-125b-2-3p and miR-933 on the activity of anticancer drugs, the MTS assay was performed in the CRC cell lines HCT116 and DLD-1. For each miRNA, specific mimics and inhibitors were used for functional experiments. The half maximal inhibitory concentrations (IC50) of anticancer drugs were calculated. As shown in Fig. 4 and Table 4, the cells with knockdown of miR-125b-2-3p were more resistant to the anticancer drugs fluorouracil, oxaliplatin and CPT-11. In addition, the cells with overexpression of miR-125b-2-3p were more sensitive to all three anticancer drugs. However, no significant change due to the anticancer drugs was found in the miR-933 knockdown or overexpression cell lines.

\section{Discussion}

Traditional clinicopathologic characteristics, such as gender, age, tumour size, tumour grade, and lymph node status, are rarely associated with the clinical response to chemotherapy of advanced CRC patients. However, the prediction of clinical response in metastatic CRC is vital for guiding therapeutic decisions. In the present study, we developed and verified a novel two-miRNA-based 


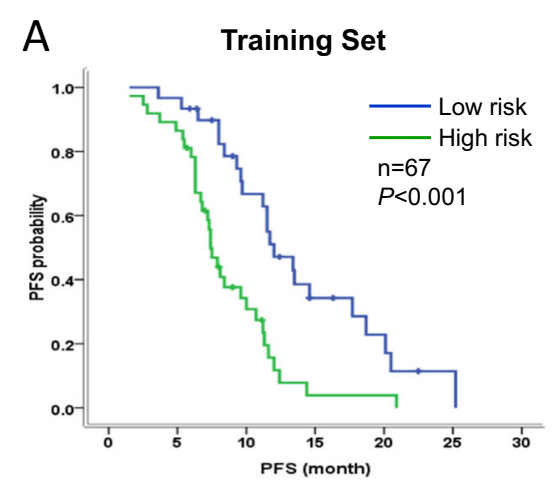

External Validation Set
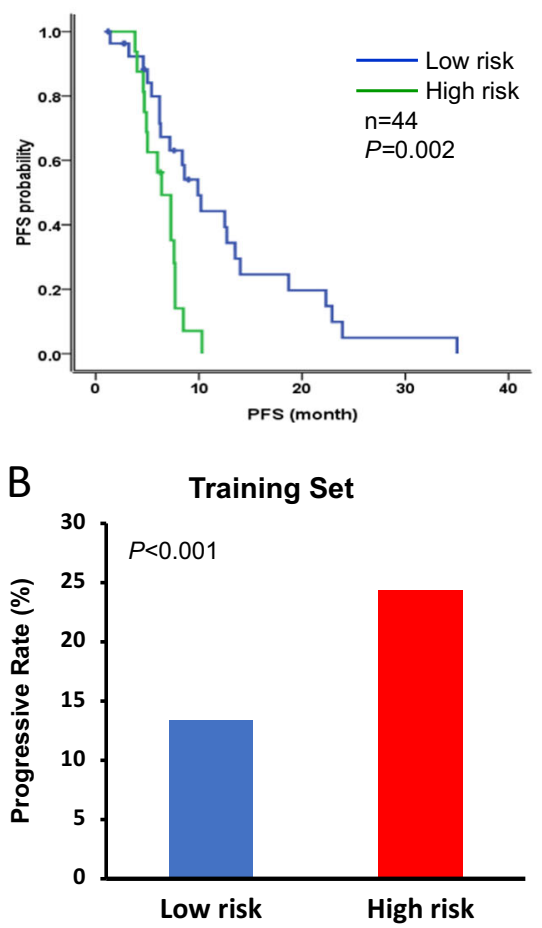

External Validation Set

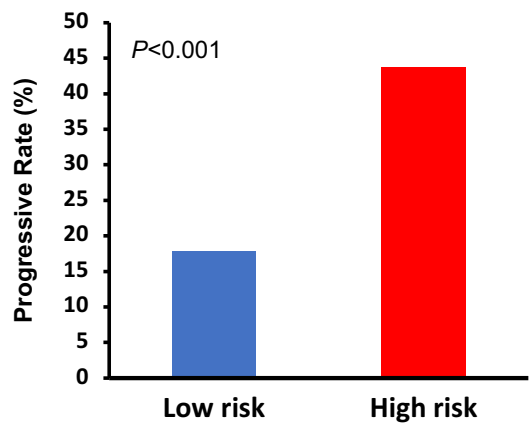

Internal Testing Set

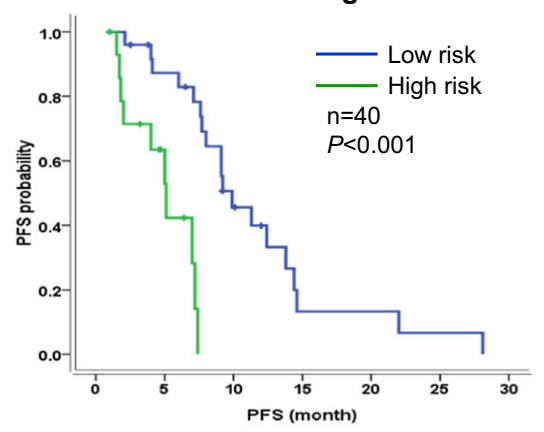

Total Dataset
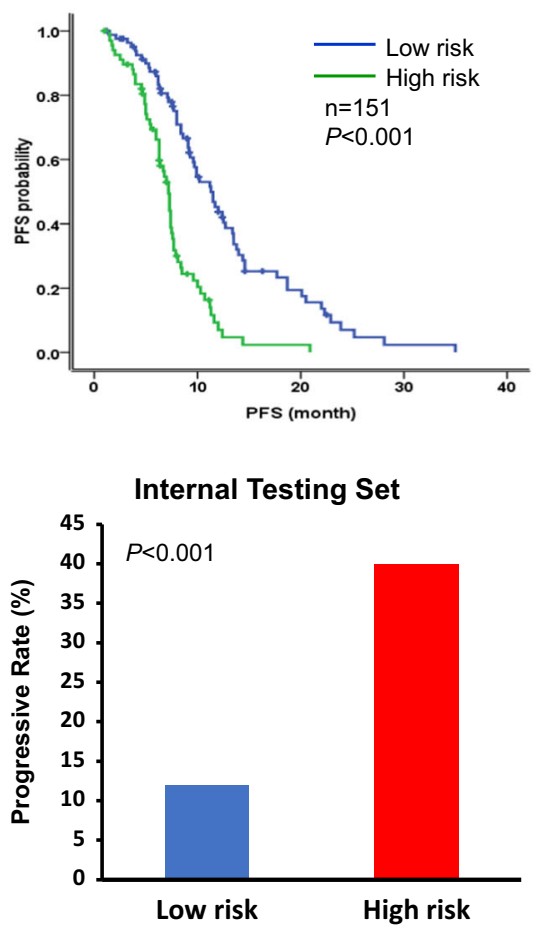

Total Dataset

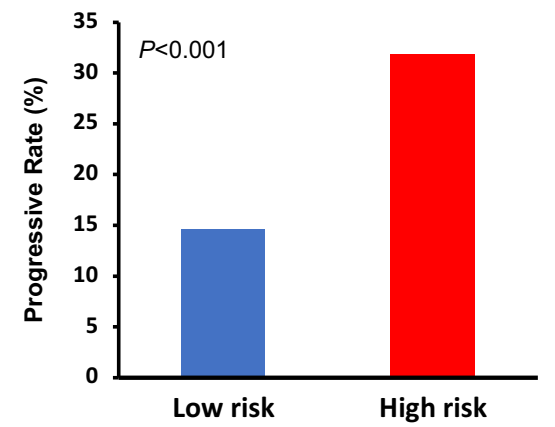

Fig. 2 Distribution of the risk score and response status to first-line chemotherapy in three cohorts and total dataset. a Kaplan-Meier survival analysis for the patients with advanced CRC according to the two-miRNA-based signature in the training set, internal testing set, external validation set and total dataset. b Progressive rate between the low-risk and high-risk groups in three cohorts and all dataset 


\section{A Training Set}

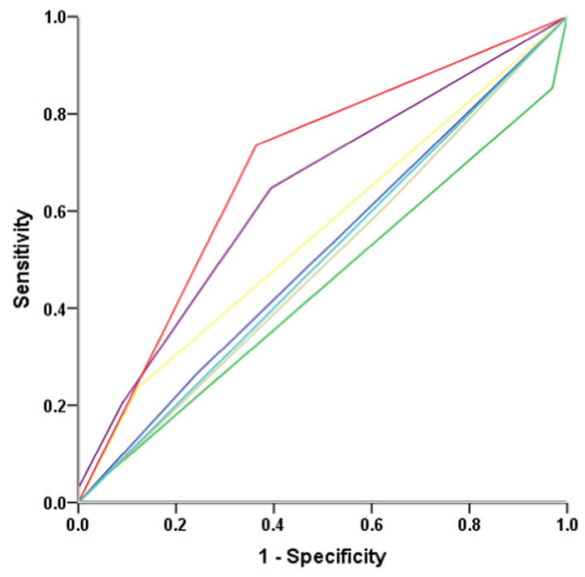

Line variable

Tumour location

Tumour grade

Metastatic location

Metastatic site num

Chemotherapy strategy

miRNA signature

\section{External Validation Set}

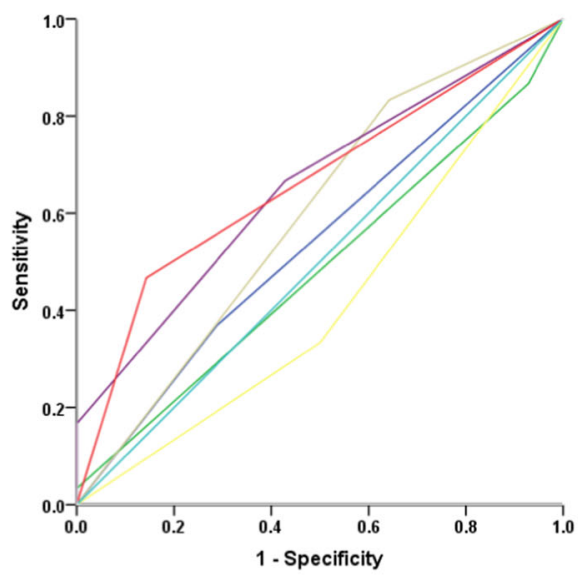

Line variable

_ Tumour location

_ Tumour grade

Metastatic location

- Metastatic site num

Chemotherapy strategy

miRNA signature
AUC $(95 \% \mathrm{Cl})$

$0.511(0.372-0.650)$

0.444(0.306-0.583)

0.491(0.351-0.630)

$0.639(0.506-0.772)$

0.557(0.419-0.695)

$0.686(0.556-0.815)$

\section{B Internal Testing Set}

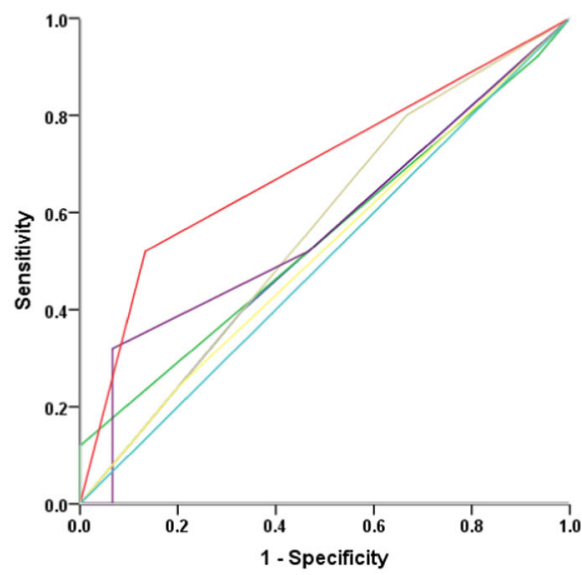

$p$-value

0.875

0.433

0.895

0.050

0.422

0.009

Line variable
Tumour location
Tumour grade
Metastatic location
Metastatic site num
Chemotherapy strategy
miRNA signature

AUC $(95 \% \mathrm{Cl})$

p-value

$0.533(0.347-0.719)$

0.549(0.369-0.730)

0.567(0.378-0.755)

0.573(0.392-0.754)

0.520(0.334-0.706)

$0.693(0.528-0.859)$

\section{Total Dataset}

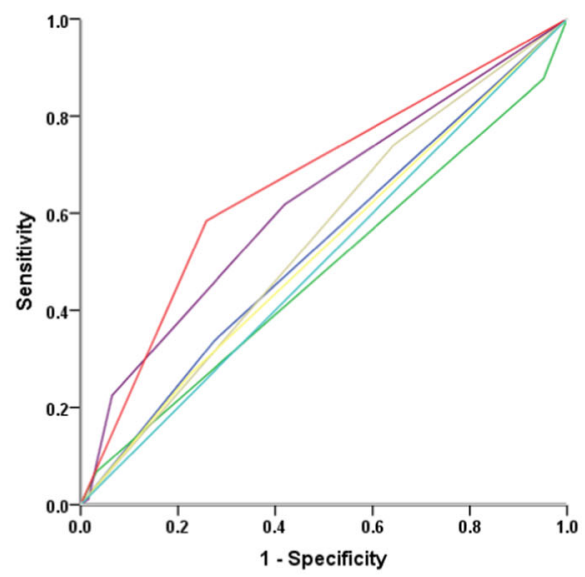

Line variable

Tumour location

AUC $(95 \% \mathrm{Cl})$

$p$-value

Tumour grade

0.531(0.438-0.625)

0.512

0.540(0.358-0.723)

0.668

0.870

0.313

0.595(0.407-0.783)

0.101

0.655(0.488-0.822)

0.378

0.417(0.232-0.601)

0.662(0.496-0.828)

0.047
$0.548(0.454-0.642) \quad 0.314$

0.625(0.536-0.714) $\quad 0.009$

0.522(0.428-0.615) $\quad 0.647$

$0.663(0.575-0.751) \quad 0.001$
$0.480(0.388-0.573) \quad 0.682$

Fig. 3 Time-dependent receiver-operating characteristic (ROC) curves for advanced CRC patients comparing the prognostic accuracy for first-line chemotherapy response by tumour location (colon vs rectum), tumour grade (low vs median/high), metastatic location (without vs with liver metastasis), metastatic site number (1 vs $\geq 2$ ), chemotherapy strategy (FOLFOX vs FOLFIRI) and the two-miRNA-based signature (high risk vs low risk) in the $\mathbf{a}$ training set, $\mathbf{b}$ internal testing set, $\mathbf{c}$ external validation set and $\mathbf{d}$ total dataset. Area under curve (AUC) was calculated and its $95 \% \mathrm{Cl}$ was estimated by SPSS 
Table 3 Interaction analysis of the signature and chemotherapy in relationship with PFS using multivariate Cox proportional hazards regression

\begin{tabular}{|c|c|c|c|c|c|c|}
\hline & \multicolumn{2}{|c|}{ Training set $(n=67)$} & \multicolumn{2}{|c|}{ Internal testing set $(n=40)$} & \multicolumn{2}{|c|}{$\begin{array}{l}\text { Independent validation } \\
\text { set }(n=44)\end{array}$} \\
\hline & $\mathrm{HR}(95 \% \mathrm{Cl})$ & $p$ & $\mathrm{HR}(95 \% \mathrm{Cl})$ & $p$ & $\mathrm{HR}(95 \% \mathrm{Cl})$ & $p$ \\
\hline Response to chemotherapy ( $C R+P R+S D$ vs PD) & $3.103(1.436-6.708)$ & 0.004 & $10.263(3.463-30.413)$ & $<0.001$ & $2.802(1.276-6.152)$ & 0.010 \\
\hline miRNA signature (low vs high risk) & $2.817(1.584-5.009)$ & $<0.001$ & $7.797(2.525-24.089)$ & $<0.001$ & $3.153(1.444-6.887)$ & 0.004 \\
\hline Interaction & $3.644(1.528-8.690)$ & 0.004 & $8.426(2.592-27.390)$ & $<0.001$ & $2.653(1.031-6.829)$ & 0.043 \\
\hline
\end{tabular}

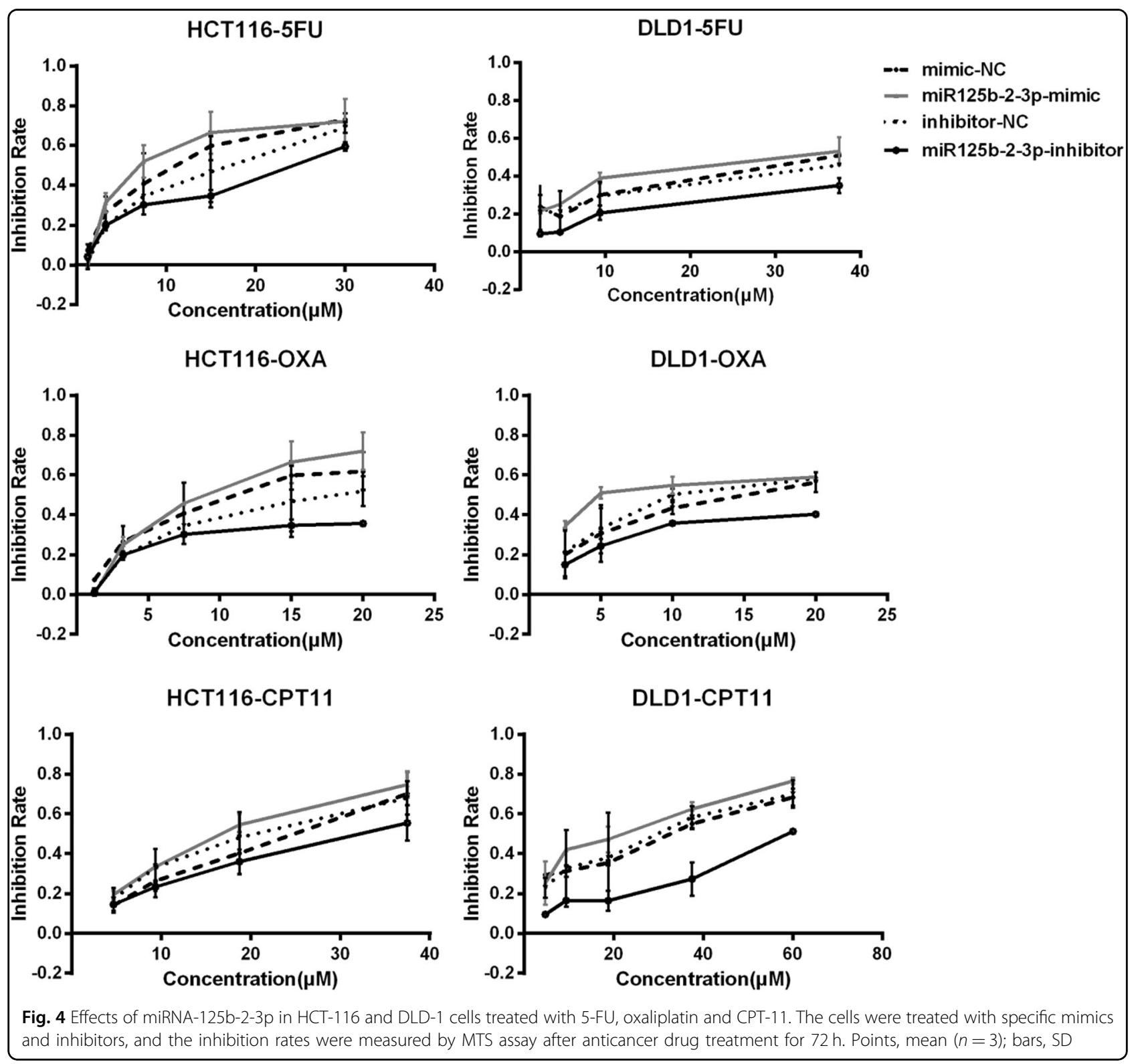


Table 4 The IC50 of anticancer drugs in CRC cell lines

\begin{tabular}{llll}
\hline & 5FU & Oxaliplatin & CPT11 \\
\hline HCT116 cell line & & & \\
mimic-NC & $9.45 \pm 1.86$ & $11.49 \pm 1.36$ & $20.81 \pm 3.18$ \\
miR933-mimic & $4.82 \pm 1.09$ & $6.77 \pm 0.74$ & $5.39 \pm 0.28$ \\
miR125b-2-3p-mimic & $6.27 \pm 0.58$ & $9.69 \pm 0.25$ & $17.58 \pm 0.59$ \\
inhibitor-NC & $14.48 \pm 3.23$ & $18.70 \pm 1.63$ & $18.37 \pm 0.25$ \\
miR933-inhibitor & $19.70 \pm 3.18$ & $26.48 \pm 1.50$ & $30.26 \pm 11.96$ \\
miR125b-2-3p-inhibitor & $25.79 \pm 1.60$ & $29.10 \pm 0.63$ & $30.92 \pm 4.40$ \\
DLD-1 cell line & & & \\
mimic-NC & $26.04 \pm 10.09$ & $13.29 \pm 1.29$ & $25.09 \pm 5.51$ \\
miR933-mimic & $18.59 \pm 8.00$ & $7.79 \pm 1.16$ & $20.28 \pm 1.39$ \\
miR125b-2-3p-mimic & $18.41 \pm 1.85$ & $9.07 \pm 1.60$ & $20.31 \pm 0.42$ \\
inhibitor-NC & $27.07 \pm 1.46$ & $15.82 \pm 1.03$ & $29.04 \pm 2.28$ \\
miR933-inhibitor & $65.49 \pm 7.57$ & $32.72 \pm 1.66$ & $48.41 \pm 1.95$ \\
miR125b-2-3p-inhibitor & $71.76 \pm 3.99$ & $50.42 \pm 9.81$ & $60.97 \pm 9.56$ \\
\hline
\end{tabular}

classifier to predict the disease progression and the benefit of receiving standard first-line chemotherapy in advanced CRC. Our results showed that this classifier could effectively assign advanced CRC patients into high- or low-risk groups with significant differences in PFS and that patients categorized as high risk based on the signature had worse survival outcomes after chemotherapy. Further use of this classifier might help identify advanced CRC patients who are most likely to benefit from first-line chemotherapy. Thus, the present miRNA signature for advanced CRC patients is a valuable prognostic and predictive tool, indicating that patients who are classified as low risk based on their risk scores have much lower chance of disease progression and have better responses to chemotherapy.

There were plenty of miRNAs which were identified differentially expressed in CRC compared with normal tissues. Among these miRNAs, two miRNAs enrolled in the signature, miR-125b and miR-933, have previously been reported as playing potential roles in cancer. MiR$125 \mathrm{~b}$ acts as either an oncogene or tumour suppressor and shows heterogenic expression in different carcinomas. For example, miR-125b is down-regulated in ovarian, bladder and breast cancer, which suggests a tumour suppressor function ${ }^{26-28}$. In contrast, miR-125b is upregulated in leukaemia and prostate cancer, where this molecule seems to act as an oncogene $e^{29,30}$. These contradictory findings also occurred in CRC. On the one hand, downregulation of miR-125b was associated with lymph node metastasis in CRC, as shown by significantly increased cell invasion, migration, and MMP activity ${ }^{31}$. On the other hand, high levels of miR-125b expression in
CRC were associated with poor survival ${ }^{32}$. In the present study, miR-125b-2-3p was down-regulated in CRC tumour tissues, and the expression of this molecule was positively correlated with PFS, which was consistent with the previous metastatic CRC study ${ }^{31}$.

Moreover, our data showed that miR-125b-2-3p affected CRC cell sensitivity to chemotherapeutic therapy. The effects of miR-125b-2-3p knockdown were more notable than those of its overexpression in CRC cells since the expression of miR-125b-2-3p was high in these two CRC cell lines. On the other hand, as we known, stromal cells play important roles in CRC biology and affect the response to chemotherapy. It was reported that the expression of miR-125b-2-3p in CRC stroma has no significant difference with that in the epithelia ${ }^{33}$. However, there was no study to compare the expression of miR$125 b-2-3 p$ in stromal cells and in cancer cells. Due to lack of microdissection procedure, our study cannot exclude the effect of stromal cells in CRC, so further study is needed to explore the role of miR-125b-2-3p in stromal cells.

In our study, the miR-933 knockdown or overexpression cells had no significant change in their response to the anticancer drugs. Furthermore, the Cox regression analysis showed that there was no significant association between the expression of miR-933 and CRC patients' PFS, which might mean that the miR-933 is not an independent prognostic and predictive biomarker in CRC.

The function and mechanism of microRNA-933 in CRC tumourigenesis, progression, and response to chemotherapy has not been thoroughly investigated. However, several studies exploring single-nucleotide polymorphisms in miR933 have shown that this molecule is associated with susceptibility to several types of human cancers ${ }^{34-36}$, although further mechanistic exploration is needed.

However, our study was a prospective observational study and not a clinical trial; therefore, there may be some inherent biases of such a study format. Moreover, the patients enrolled in the present study were all Chinese individuals whose clinical characteristics might be different from those of other ethnic groups; thus, the generalizability of this study might be limited. Clearly, an openlabel, multicentre, randomized clinical trial is needed to further validate this novel signature. Furthermore, the mechanism for how miR-125b-2-3p affects CRC sensitivity to chemotherapy warrants further exploration.

In summary, the present study shows that the twomiRNA signature can successfully classify advanced CRC patients into groups with high and low risks of tumour progression. In addition, this signature might be a reliable prognostic and predictive tool for tumour progression in patients with advanced CRC and might be able to predict the benefit of receiving standard first-line chemotherapy in CRC. 


\section{Materials and methods}

\section{Patients and clinical database}

This prospective observational study was started in 2008. A total of 151 patients with histopathologically and clinically diagnosed advanced CRC from 1 January 2008 to 30 June 2014 were enrolled in this project. The following were the criteria for enrolment: (1) patients diagnosed with advanced CRC; (2) histologically confirmed $\mathrm{CRC}$ with at least one measurable lesion as defined by the Response Evaluation Criteria in Solid Tumours; (3) patients who received National Comprehensive Cancer Network (NCCN)-guided first-line chemotherapy (FOLFOX or FOLFIRI strategy); (4) patients with completed clinicopathological information and follow-up data; (5) patients have not received previous chemotherapy for the target lesions, however, patients who had received adjuvant/neoadjuvant chemotherapy were enrolled if the interval was more than 12 months after the end of chemotherapy; and (6) sufficient formalin-fixed paraffinembedded (FFPE) tissues for further study. Prior to primary chemotherapy, tumour samples were acquired during palliative operations or colonoscopy biopsies and embedded in paraffin.

For the training and internal testing sets, 107 metastasis CRC patients treated at Sun Yat-sen University Cancer Center (SYSUCC, Guangzhou, China) were included from 1 January 2008 to 30 June 2014. These 107 patients were randomly divided into training and internal testing sets (67 and 40 patients, respectively). To verify the prognostic or predictive accuracy of the signature, we included another 44 advanced CRC patients as the independent external validation cohort from the Foshan First People's Hospital (FFPH), Foshan, between 1 December 2008 to 31 December 2013. The same inclusion and exclusion criteria were used. Follow-up procedure for these patients was finished on 31 July 2016. The median follow-up time was 44.6 month. Additionally, to obtain the miRNA expression profiles, 21 frozen tumour and paired adjacent normal mucosa samples from metastatic CRC patients obtained at SYSUCC from 1 January 2003 to 31 December 2005 were used.

The clinical and clinicopathological classification and stage were clarified according to the American Joint Committee on Cancer (AJCC) criteria. Prior to carrying out the research, this project was approved by the Institutional Research Ethics Committee and all the participants signed informed consent forms. All the patients received tumour assessments every 6 weeks during therapy and at the end of treatment. PFS was defined as the period between the initiation treatment of first-line chemotherapy and the date of disease progression or death. The blinded endpoint study was not applicable in this project. The study was followed REMARK guideline (supplementary Table 1).

\section{Sample preparation}

The FFPE tissue samples were composed of at least $80 \%$ tumour cells. Total RNA was isolated from 151 metastatic CRC samples and 21 paired CRC tumour and adjacent normal tissues as previously described ${ }^{37}$. The purity and concentration of RNA were quantified by using a Nanodrop 2000 spectrophotometer (Thermo Fisher Scientific, USA).

RNA labelling, hybridization and array scanning were carried out as previously described and performed by CapitalBio (Capital-Bio Corp., Beijing, China) ${ }^{38}$. Briefly, total RNA was purified and concentrated using the mirVana $^{\mathrm{Tm}}$ miRNA Isolation Kit (Ambion, Austin, TX, USA). Purified RNA was labelled with fluorescein Cy3 and hybridization was conducted using an Agilent Human miRNA Microarray (miRBase release 19.0). The fluorescence intensities were converted into digital data and Log2 transformed using Feature Extraction (version 10.7). Differential gene expression was analysed by using GeneSpring software version 12.0 (Agilent, USA). The miRNAs were categorized as significantly differentially expressed if the $p$ value was lower than 0.05. Heat maps were formed using the Cluster 3.0 package software. Microarray data were deposited into the Gene Expression Omnibus (GEO) database of the National Center for Biotechnology Information (NCBI) (accession no. GSE108153).

MiRNA expression was detected by qRT-PCR in the $151 \mathrm{CRC}$ samples and 21 paired CRC and adjacent normal tissues. Total RNA was obtained with TRIzol reagent (Life Technologies, Carlsbad, California, USA), and $2 \mu \mathrm{g}$ total RNA was reverse transcribed using the All-in-One ${ }^{\text {тM }}$ miRNA First-Strand cDNA Synthesis Kit (GeneCopoeia Inc., USA). The All-in-One ${ }^{\mathrm{Tm}}$ miRNA qPCR Kit (GeneCopoeia Inc., USA) and Roche Lightcycler 480 instrument (Roche Diagnostics, Basel, Switzerland) were used for qRT-PCR analysis. All experiments were conducted according to the manufacturer's instructions. The results were normalized to U48 expression and analysed using in the $2-\Delta$ ct method. The primers were synthesized by GeneCopoeia, Inc.

\section{Identification of candidate miRNAs and development of the miRNA signature}

We performed the miRNA microarray on 21 paired CRC cancer and adjacent normal frozen tissues samples. We identified the differentially expressed miRNAs using the significance analysis of microarrays with a false discovery rate $(\mathrm{FDR})<0.05$. By combining the RNA expression data from the microarray and follow-up data, we obtained 33 significantly differentially expressed miRNA candidates correlated with PFS by Cox regression analysis $(p<0.1)$.

Differential miRNA expression was examined by qRT$\mathrm{PCR}$ in the training set and Cox proportional hazard 
regression modelling was then performed to analyse the correlation between RNA expression and PFS data in patients. The miRNA signature was validated in the internal and external validation sets. The prognostic or predictive accuracy of each feature and the miRNA-based signature was investigated by time-dependent receiver operating characteristic (ROC) analysis, which was measured by the area under the curve.

\section{LASSO bagging variable selection}

To determine the miRNAs most predictive of a high/ low recurrence risk signature, we utilized the LASSO bagging strategy. At the data preparation step, we first obtained 33 candidate markers from the differential expression and uni-variable Cox regression analysis described above, and we then examined their RNA abundance in a training data set of 67 cancer samples using RT-PCR. Since the relative RT-PCR values of miRNA in each sample were too small to manage, we applied logarithmic transformation ( 2 bases) on 10,000 multiplied matrices to normalize the values into a readable range. As a result, a matrix of 33 variables and 67 data points (samples) was built for further LASSO bagging analysis, the procedure for which is described as follows: (1) the data points were resampled 1000 times with replacement to generate 1000 training matrices. (2) For each matrix with recurrent survival outcomes, we performed LASSO Cox regression analysis using 10-fold cross validation. The tuning parameter $\lambda$ was selected by 1-SE (standard error), and we finally obtained a list of variables that had beta-coefficients different than zero in LASSO. (3) All variable lists obtained in each resample were combined to calculate the resample model inclusion proportion (RMIP) for each miRNA (also explained by an observed frequency in 1000 resamples). (4) The RMIP was used as the weight of each variable, and the top two miRNAs were selected as candidate markers for building the signature. All running analysis scripts were programmed with R (v3.3.1), and LASSO was adopted from the glmnet package (2.0-10).

\section{Cell culture}

The HCT116 and DLD1 CRC cell lines were purchased from ATCC (Manassas, VA, USA) and cultured in RPMI1640 basic medium $(1 \times)$ with $10 \%$ foetal bovine serum (Thermo Fisher Scientific, Carlsbad, California, USA) at $37^{\circ} \mathrm{C}$ with $5 \% \mathrm{CO}_{2}$. All cell lines were tested and authenticated by short tandem repeat DNA testing at Beijing Microread Gene Tech. Co., Ltd. (Beijing, China) prior to use.

\section{MTS assay}

Cells were seeded onto 96-well plates at a density of $3 \times$ $10^{3}$ cells/well and incubated with irinotecan, fluorouracil and oxaliplatin (Selleck, Houston, TX, USA) for $72 \mathrm{~h}$. Then, the cells were stained with $20 \mu \mathrm{l}$ of sterile MTS (3-(4, 5-dimethylthiazol-2-yl) -5- (3-carboxymethoxyphenyl) -2- (4-sulfophenyl)-2H-tetrazolium) dye (Promega, Madison, WI, USA) for $2 \mathrm{~h}$ at $37^{\circ} \mathrm{C}$. The absorbance was measured at a wavelength of $490 \mathrm{~nm}$. All experiments were conducted in triplicate.

\section{Statistical analysis}

Student's $t$ test was used to compare continuous variables between paired tumour and adjacent normal tissues. For survival analyses, the curves were plotted with the Kaplan-Meier method, and the different groups were compared using the log-rank test. Adjusted hazard ratios (HRs) with 95\% confidence intervals (CI) were calculated using Cox proportional hazards modelling. The median value of the risk score in the training set was taken as the cut-off value. Post hoc statistical power analysis was calculated to evaluate the study sample size. All statistical tests were two-sided and considered significant at twosided $p$ values $<0.05$, unless specifically stated. All the data analyses above were performed using SPSS version 22.0 statistical software or R3.1.2 (R Foundation for Statistical Computing) software.

\section{Acknowledgements \\ This work was supported by the National Natural Science Foundation of China (81572392); the National Key Research and Development Program of China (2018YFC1313300, 2016YFC1201704); Tip-top Scientific and Technical Innovative Youth Talents of Guangdong special support program (2016TQ03R614); the Natural Science Foundation of Guangdong Province (2014A030312015); the Science and Technology Program of Guangdong (2015B020232008); and the Science and Technology Program of Guangzhou (15570006, 158100066, 201400000004-5, 201508020250, 201604020003, 201604020005); Pearl River Nova Program of Guangzhou (201610010068).}

\section{Author details}

${ }^{1}$ State Key Laboratory of Oncology in South China, Collaborative Innovation Center for Cancer Medicine, Sun Yat-sen University Cancer Center, 510060 Guangzhou, China. 'Foshan First People's Hospital, 528000 Foshan, China. ${ }^{3}$ The Third Affiliated Hospital, Sun Yat-sen University, 510630 Guangzhou, China

\begin{abstract}
Author contributions
Jia-huan Lu: Acquisition of data and experiments set-up; Zhi-xiang Zuo, Qi Zhao: Data analysis and interpretation; Wei Wang, Miao-zhen Qiu, Hui-yan Luo, Feng Wang, Xiao-li Wei: Samples collection; Zhan-hong Chen, Hai-yu Mo, Qinian Wu: Acquisition of follow-up data and survival statistical analysis; Dongdong Yang, Yun Wang: Acquisition of data; Huai-qiang Ju: Experiments strategies/trouble shooting; Rui-hua Xu: Study design, manuscript editing of the article; Zhao-lei Zeng: Conception and design, Manuscript writing, financial support.
\end{abstract}

Conflict of interest

The authors declare that they have no conflict of interest.

\section{Publisher's note}

Springer Nature remains neutral with regard to jurisdictional claims in published maps and institutional affiliations.

The online version of this article (https://doi.org/10.1038/s41420-018-0133-7) contains supplementary material, which is available to authorized users. 
Received: 18 October 2018 Revised: 22 November 2018 Accepted: 3 December 2018

Published online: 18 December 2018

\section{References}

1. Siegel, R. L., Miller, K. D. \& Jemal, A. Cancer Statistics, 2017. CA Cancer J. Clin. 67, 7-30 (2017).

2. Siegel, R. L., et al. Colorectal cancer statistics, 2017. CA Cancer J. Clin. 67, 177-193 (2017).

3. Torre, L. A. et al. Global cancer statistics, 2012. CA Cancer J. Clin. 65, 87-108 (2015).

4. Edwards, M. S., Chadda, S. D., Zhao, Z., Barber, B. L. \& Sykes, D. P. A systematic review of treatment guidelines for metastatic colorectal cancer. Colorectal Dis. 14, e31-e47 (2012).

5. Ferlay, J. et al. Cancer incidence and mortality worldwide: sources, methods and major patterns in GLOBOCAN 2012. Int. J. Cancer J. Int. du Cancer 136, E359-E386 (2015).

6. Luo, H. Y. et al. Single-agent capecitabine as maintenance therapy after induction of XELOX (or FOLFOX) in first-line treatment of metastatic colorectal cancer: randomized clinical trial of efficacy and safety. Ann. Oncol. 27, 1074-1081 (2016)

7. Hurwitz, H. et al. Bevacizumab plus irinotecan, fluorouracil, and leucovorin for metastatic colorectal cancer. N. Engl. J. Med. 350, 2335-2342 (2004).

8. Tol, J. et al. Chemotherapy, bevacizumab, and cetuximab in metastatic colorectal cancer. N. Engl. J. Med. 360, 563-572 (2009).

9. Venook, A. P. et al. Effect of first-line chemotherapy combined with cetuximab or bevacizumab on overall survival in patients with KRAS wild-type advanced or metastatic colorectal cancer: a randomized clinical trial. JAMA 317, 2392-2401 (2017).

10. He, L. \& Hannon, G. J. MicroRNAs: small RNAs with a big role in gene regulation. Nat. Rev. Genet. 5, 522-531 (2004).

11. Hayes, J., Peruzzi, P. P. \& Lawler, S. MicroRNAs in cancer: biomarkers, functions and therapy. Trends Mol. Med. 20, 460-469 (2014).

12. Bartel, D. P. MicroRNAs: target recognition and regulatory functions. Cell 136, 215-233 (2009).

13. Mohr, A. M. \& Mott, J. L. Overview of microRNA biology. Semin. Liver. Dis. 35, 3-11 (2015).

14. Sun, Y. et al. Key nodes of a microRNA network associated with the integrated mesenchymal subtype of high-grade serous ovarian cancer. Chin. J. Cancer 34, 28-40 (2015).

15. Croce, C. M. Causes and consequences of microRNA dysregulation in cancer. Nat. Rev. Genet. 10, 704-714 (2009).

16. Esquela-Kerscher, A. \& Slack, F. J. Oncomirs - microRNAs with a role in cancer. Nat. Rev. Cancer 6, 259-269 (2006).

17. Garzon, R., Marcucci, G. \& Croce, C. M. Targeting microRNAs in cancer: rationale, strategies and challenges. Nat. Rev. Drug. Discov. 9, 775-789 (2010).

18. Iorio, M. V. \& Croce, C. M. MicroRNA dysregulation in cancer: diagnostics, monitoring and therapeutics. A comprehensive review. EMBO Mol. Med. 9, 852 (2017).
19. Yu, X., Li, Z., Yu, J., Chan, M. T. \& Wu, W. K. MicroRNAs predict and modulate responses to chemotherapy in colorectal cancer. Cell. Prolif. 48, 503-510 (2015).

20. Diaz, T. et al. Role of miR-200 family members in survival of colorectal cancer patients treated with fluoropyrimidines. J. Surg. Oncol. 109, 676-683 (2014).

21. Nakajima, G. et al. Non-coding MicroRNAs hsa-let-7g and hsa-miR-181b are Associated with Chemoresponse to S-1 in Colon Cancer. Cancer Genom. Proteom. 3, 317-324 (2006).

22. Luo, X., Burwinkel, B., Tao, S. \& Brenner, H. MicroRNA signatures: novel biomarker for colorectal cancer? Cancer Epidemiol. Biomark. Prev. 20, 1272-1286 (2011).

23. Zhang, J. X. et al. Prognostic and predictive value of a microRNA signature in stage II colon cancer: a microRNA expression analysis. Lancet Oncol. 14, 1295-1306 (2013).

24. Wu, T. T., Chen, Y. F., Hastie, T., Sobel, E. \& Lange, K. Genome-wide association analysis by lasso penalized logistic regression. Bioinformatics 25, 714-721 (2009).

25. Gilhodes, J. et al. Comparison of variable selection methods for highdimensional survival data with competing events. Comput. Biol. Med. 91, 159-167 (2017).

26. Nam, E. J. et al. MicroRNA expression profiles in serous ovarian carcinoma. Clin. Cancer Res. 14, 2690-2695 (2008).

27. Iorio, M. V. et al. MicroRNA gene expression deregulation in human breast cancer. Cancer Res. 65, 7065-7070 (2005).

28. Ichimi, T. et al. Identification of novel microRNA targets based on microRNA signatures in bladder cancer. Int. J. Cancer 125, 345-352 (2009).

29. Chaudhuri, A. A. et al. Oncomir miR-125b regulates hematopoiesis by targeting the gene Lin28A. Proc. Natl Acad. Sci. USA 109, 4233-4238 (2012).

30. Pang, Y., Young, C. Y. \& Yuan, H. MicroRNAs and prostate cancer. Acta Biochim. Biophys. Sin. 42, 363-369 (2010).

31. Fujino, Y. et al. Downregulation of microRNA-100/microRNA-125b is associated with lymph node metastasis in early colorectal cancer with submucosal invasion. Cancer Sci. 108, 390-397 (2017).

32. Nishida, N. et al. MicroRNA miR-125b is a prognostic marker in human colorectal cancer. Int. J. Oncol. 38, 1437-1443 (2011).

33. Zhou, X. G. et al. Identifying miRNA and gene modules of colon cancer associated with pathological stage by weighted gene co-expression network analysis. Onco. Targets Ther. 11, 2815-2830 (2018).

34. Zhang, Y. et al. Association of microRNA-933 variant with the susceptibility to gastric cancer. J. BUON 22, 390-395 (2017).

35. Wei, W. J. et al. Association study of single nucleotide polymorphisms in mature microRNAs and the risk of thyroid tumor in a Chinese population. Endocrine 49, 436-444 (2015).

36. Dong, G., Zhang, R., Xu, J. \& Guo, Y. Association between microRNA polymorphisms and papillary thyroid cancer susceptibility. Int. J. Clin. Exp. Pathol. 8, 13450-13457 (2015)

37. Tan, S. C. \& Yiap, B. C. DNA, RNA, and protein extraction: the past and the present. J. Biomed. Biotechnol. 2009, 574398 (2009).

38. Guo, S. T. et al. MicroRNA-497 targets insulin-like growth factor 1 receptor and has a tumour suppressive role in human colorectal cancer. Oncogene $\mathbf{3 2}$ 1910-1920 (2013). 